\title{
Leveraging Health-Related Quality of Life in Population Health Management: The Case for Healthy Days
}

\author{
S. Lane Slabaugh, PharmD, MBA, Mona Shah, MPH, PhD, Matthew Zack, MD, MPH, \\ Laura Happe, PharmD, MPH, Tristan Cordier, MPH, Eric Havens, MA, \\ Evan Davidson, MD, ${ }^{1}$ Michael Miao, MD, ${ }^{1}$ Todd Prewitt, MD, and Haomiao Jia, PhD ${ }^{4,5}$
}

\begin{abstract}
Measuring population health with morbidity and mortality data, often collected at the site of care, fails to capture the individual's perspective on health and well-being. Because health happens outside the walls of medical facilities, a holistic and singular measure of health that can easily be captured for an entire population could aid in understanding the well-being of communities. This paper postulates that Healthy Days, a health-related quality of life measure developed and validated by the Centers for Disease Control and Prevention, is an ideal survey instrument to advance population health. A systematic literature review was conducted and revealed a strong evidence base using Healthy Days with significant correlations to chronic disease conditions. Building on the literature base and experience, methods for analyzing Healthy Days data are discussed, including stratified sampling techniques, statistical measures to account for variance, and modeling techniques for skewed distributions. Using such analytic techniques, Healthy Days has been used extensively in national health surveillance. As the health care system faces increasing costs and constrained resources, the Healthy Days survey instrument can be used to inform public policies and allocate health service resources. Because Healthy Days captures broad dimensions of health from the individual's perspective, it is a simple way to holistically measure the health and well-being of a population and its trend over time. Expanded use of Healthy Days can aid population health managers and contribute to the understanding of the broader determinants of the nation's and individual community's health and aid in evaluating progress toward health goals.
\end{abstract}

\section{Introduction}

$\mathbf{S}_{\mathrm{h}}^{\mathrm{E}}$ EVERAL DIFFERENT GROUPS ARE ACCOUNTABLE for the health and medical care of communities. Some, such as the Indian Health Service, have wide responsibility to provide all health programs for a distinct population. Most, however, manage only certain aspects of health programs or work within less defined communities. For example, state or local health departments greatly influence public health initiatives but generally are not responsible for directly providing health care. Health care providers, on the other hand, provide medical care to their patients but usually cannot affect social determinants of health in their communities. Given this fragmented system of responsibility for overall health in the United States, it can be difficult for any of these groups that are accountable for community medical care to get a complete look at the health of their community. ${ }^{1,2}$

Traditional measures of health, such as morbidity and mortality, are important health indicators but fall short of incorporating the voice of individuals. Health-related quality of life (HRQOL) — a multidimensional concept encompassing physical, mental, emotional, and social functioning-is

\footnotetext{
${ }^{1}$ Humana Inc., Louisville, Kentucky.

${ }^{2}$ Robert Wood Johnson Foundation, Princeton, New Jersey.

${ }^{3}$ Centers for Disease Control and Prevention, Atlanta, Georgia.

${ }^{4}$ Mailman School of Public Health, Columbia University, New York, New York.

${ }^{5}$ School of Nursing, Columbia University, New York, New York.
}

(C) Slabaugh et al. 2016; Published by Mary Ann Liebert, Inc. This Open Access article is distributed under the terms of the Creative Commons Attribution Noncommercial License (http://creativecommons.org/licenses/by-nc/4.0/) which permits any noncommercial use, distribution, and reproduction in any medium, provided the original author(s) and the source are credited. 
widely accepted as a valid measure of the burden of disease as experienced directly by individuals, providing a more holistic view of overall health. ${ }^{3-5}$ Furthermore, HRQOL is highly correlated with traditional measures of health, including morbidity, mortality, and health care costs. ${ }^{6-10}$

Given its multidimensional nature, HRQOL is an important measure of population health. ${ }^{3}$ When applied to a population, HRQOL assessment provides the global picture that is missing from the somewhat limited perspective of various agencies and professionals. HRQOL is one of the 4 Foundation Health Measures used by Healthy People 2020 to assess the health of communities. ${ }^{11}$ HRQOL can be used to measure health disparities, track the influence of social determinants on overall health, and shed light on the ultimate impact of the health care system. However, it can be challenging to select the right HRQOL instrument. Although several available instruments have demonstrated validity and reliability, they vary in scope, intended purpose, applicability, and general ease of use. To measure HRQOL, the ideal instrument for those who manage population health would be holistic and easy to administer, and would measure the individual's perspective while being understandable to health care providers and the general public alike.

\section{Healthy Days}

During the late 1980s, the Centers for Disease Control and Prevention (CDC) sought to develop a survey instrument to capture self-perceived HRQOL in a short questionnaire that was easy for people to understand and answer. The resulting tool-the CDC-HRQOL-4, or "Healthy Days" — consists of 4 questions that ask people about how they perceive their own recent health (Table 1). Answers to the second question about recent physical health and the third question about recent mental health provide a summary index of unhealthy days (UHD) for an individual. When a respondent replies with zero days to questions 2 and 3, the respondent is not asked the final question on activity limitation, which is imputed as zero.

The CDC developed this questionnaire as an alternative to other, longer HRQOL measures for large population-based surveys; it is easily understood by the public and policy makers. Its value lies in its simplicity. It is also unique in that it measures physical and mental health as separate domains. Starting in 1993, Healthy Days was incorporated into the state-based Behavioral Risk Factor Surveillance System (BRFSS), a continuous, statebased, random telephone survey of community-dwelling US adults aged 18 and older. The inclusion of Healthy Days in the BRFSS survey has become one of the greatest strengths of the instrument, as this survey provides health-related information for a multi-year sample across the US population. A response rate of $>98 \%$ to the Healthy Days questions demonstrates that these questions are easy for people to answer. ${ }^{12}$ No other measures of HRQOL, such as the Medical Outcomes Study Short-forms (SF12 and SF-36) or the EuroQoL Group's EQ-5D, are available in a similarly large annual sample as in the BRFSS, which tracks trends over time and in different geographic areas. Another national survey, the National Health and Nutrition Examination Survey (NHANES), also included the Healthy Days questions from 2000 through 2011, and the Medicare Health Outcomes Survey has included them since 2003.

In 2000 the CDC National Center for Chronic Disease Prevention and Health Promotion's Division of Adult and Community Health published a report, Measuring Healthy Days: Population Assessment of Health-Related Quality of Life. ${ }^{11}$ This report describes the aims of the Healthy Days survey instrument and the research that has demonstrated its validity and reliability. This research shows that the questions have high construct validity (do a good job of measuring the intended concepts) and concurrent validity (its results align with other validated instruments that measure the same concepts). The validity of Healthy Days has been confirmed across elderly, adolescent, and disabled population segments, as well as in specific clinical populations such as those with spinal cord injuries. ${ }^{9,13-17}$ Furthermore, the Healthy Days questions are a valid and reliable measure of population health in distinct geographic areas including Canada and Puerto Rico, as well as at the state and local level within the United States. ${ }^{18-20}$ Healthy Days was recently suggested as a potential measure of population health within the Triple Aim (population health, patient experience, and per capita cost). ${ }^{21}$

Given its demonstrated validity and reliability, its unique simplicity, and its ability to reflect people's perceptions of their own health, Healthy Days can be an effective metric of overall health for those who manage the health of population groups, whether an organization's responsibilities involve community interventions, disease management, or delivery of clinical care. This paper will review the existing published research using Healthy Days as a measure of HRQOL, describe practical considerations in collection and analysis of the data, and show how the survey instrument can be used to inform public policy.

\section{Use of Healthy Days in the Literature}

To quantify the scope and current use of the Healthy Days survey instrument, a systematic literature review was conducted using the PubMed and Google Scholar databases and the following search terms Healthy Days, Unhealthy Days, HRQOL-4, and the 4 questions. The search was limited to English, and no constraints were placed on publication date. Two independent reviewers screened the publications retrieved and excluded publications of study populations younger than 18 years of age or in other than North American

Table 1. CDC-HRQOL-4 Survey Instrument - Healthy Days

$1 \quad$ Would you say that in general your health is excellent, very good, good, fair, or poor?

2 Now thinking about your physical health, which includes physical illness and injury, for how many days during the past 30 days was your physical health not good?

3 Now thinking about your mental health, which includes stress, depression, and problems with emotions, for how many days during the past 30 days was your mental health not good?

4 During the past 30 days, for about how many days did poor physical or mental health keep you from doing your usual activities, such as self-care, work, or recreation?

$\mathrm{CDC}-\mathrm{HRQOL}=\mathrm{Centers}$ for Disease Control and Prevention Health-Related Quality of Life. 
populations, method or review studies, non-peer-reviewed studies, and non-Healthy Days-related studies. The reviewers assessed the included studies for their characteristics and classified them into studies of specific diseases, general health, prevention, and socioeconomic characteristics.

This search strategy identified 211 studies, 110 of which met the inclusion criteria (see online Appendix A, available in the online article at www.liebertpub.com/pop). Ninety-one studies were excluded for the following reason(s): The study focused on populations younger than 18 years of age $(n=10)$; the study focused on other than North American populations $(n=28)$; the study focused only on methods or was a review $(n=18)$; the study was not peer reviewed $(n=12)$; or the study was unrelated to Healthy Days measurements $(n=23)$ (Fig. 1).

Of the 110 studies included in the review, the strongest similarity among the articles was that most $(80.9 \%, \mathrm{n}=89)$ used Healthy Days data from a secondary data source, most commonly BRFSS $(n=66)$, the NHANES $(n=7)$, and the California Health Interview Survey $(n=4)$. Only $19.1 \%$ $(\mathrm{n}=21)$ of the studies collected Healthy Days as primary data for their studies. Most $(53.6 \%, \mathrm{n}=59)$ of the studies evaluated Healthy Days in the context of a specific disease state, including mental disorders $(n=12)$, diabetes $(n=8)$, arthritis $(n=7)$, cardiovascular disease $(n=6)$, obesity $(n=6)$, and pulmonary disease $(n=5)$. General health studies assessing HRQOL vs. mortality or covering multiple chronic conditions accounted for $21.8 \%(n=24)$ of the included studies, followed by socioeconomic characteristics $(13.6 \%, \mathrm{n}=15)$ and lifestyle factors $(10.9 \%, \mathrm{n}=12)$. There were no observed trends geographically, as only California $(n=5)$ and North Carolina $(n=5)$ had 5 or more regionally focused studies.

How the studies reported UHD varied by the type of study. The studies that evaluated specific health conditions usually contrasted the mean numbers of physically UHD and mentally UHD for people with the condition to those for people without the condition (Table 2). Although less common, several studies summed the number of physically and mentally UHD, with a maximum of 30 days, to report total UHD. Other studies converted the number of UHD into a binary variable using a threshold of 14 UHD. This 14-day threshold was first reported in the late 1990 s as a way to analyze the third Healthy Day question on mental distress. Fourteen days, originally selected because clinicians and clinical researchers had used a similar duration as a marker for depression and anxiety disorders, is now a generally accepted threshold used by the CDC for

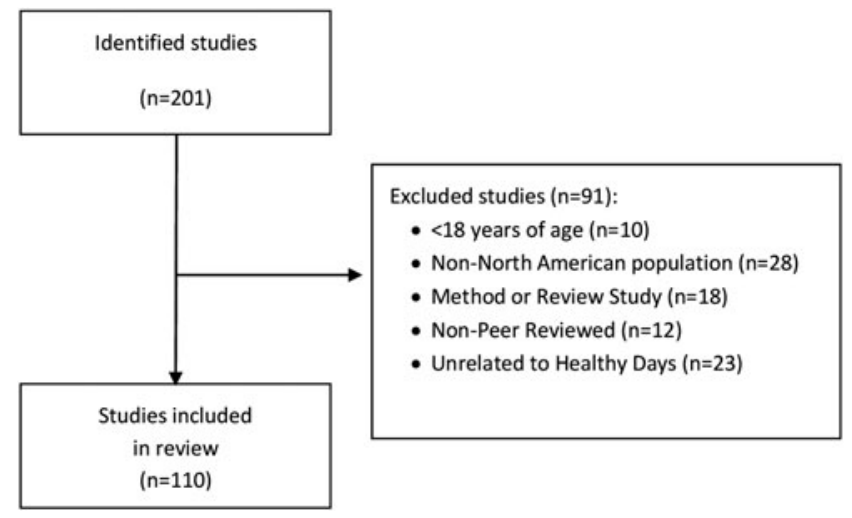

FIG. 1. Study attribution table. longitudinal tracking and ranking states. ${ }^{5,11,22-23}$ Finally, several studies used multivariate regression modeling to understand the association between disease states and having 14 or more UHD. Although these reporting consistencies allow for comparisons between studies, such comparisons require caution to account for baseline differences in the study populations and the methods of statistical analyses.

Besides reporting UHD from the second and third questions on the survey instrument, $72 \%(n=79)$ of the studies reported the responses to the fourth question, which addresses activity limitations. The CDC describes question 4 as a "global indicator of perceived disability as well as an indicator of productive human capital,"11 so this finding underscores the importance of self-reported activity limitations and functional status among those researching HRQOL.

Many studies used responses to the third Healthy Days question on mental health to calculate self-reported frequent mental distress (FMD), defined as 14 or more mentally UHD within the last 30 days. Of the articles evaluating mental disorders, 6 measured FMD while the others either dichotomized or reported mean mentally UHD. In one study, for example, people who reported activity limitation related to physical, mental, or emotional problems were more than twice as likely to have FMD (adjusted odds ratio $=2.59$, $95 \%$ confidence interval $[\mathrm{CI}], 2.33-2.87) .^{24}$

This systematic review reveals strong literature support for using Healthy Days to measure HRQOL among comparative populations, including those with and without health conditions. Most of the studies relied on secondary analysis of existing data, suggesting that barriers may exist to primary collection of Healthy Days data. As the CDC itself is the primary producer of research studies using Healthy Days, there is substantial opportunity for expanded research contributions.

\section{Practical Considerations for Data Collection and Analysis of Healthy Days}

CMS and other payers typically draw from large populations when conducting Healthy Days surveys. Collecting data from the entire population of interest is ideal but can be resource-intensive. Sample surveys provide an alternative for estimating Healthy Days across a population. Different techniques can be used to estimate the sample size needed to generate precise estimates and detect small differences (eg, $\leq 5 \%$ absolute difference). Because of the large variance associated with the highly skewed distribution of the Healthy Days metric, large survey samples are needed, particularly if the underlying population is large.

An approach that randomly samples different subpopulations separately, yielding a stratified random sample, may be used to assure that the study sample includes sufficient numbers of participants in each stratum, or subgroup, to allow separate estimates at the desired level of precision. For instance, private payer organizations may wish to sample and analyze according to participation in Medicare, Medicaid, Affordable Care Act exchanges, and/or employer plans. Subgroup analysis acknowledges the inherent and substantial demographic, behavioral, and clinical differences between subpopulations. However, stratified random sampling may result in oversampling of some subpopulations (ie, recruitment of participants in proportions that do not reflect the population distribution of characteristics). 


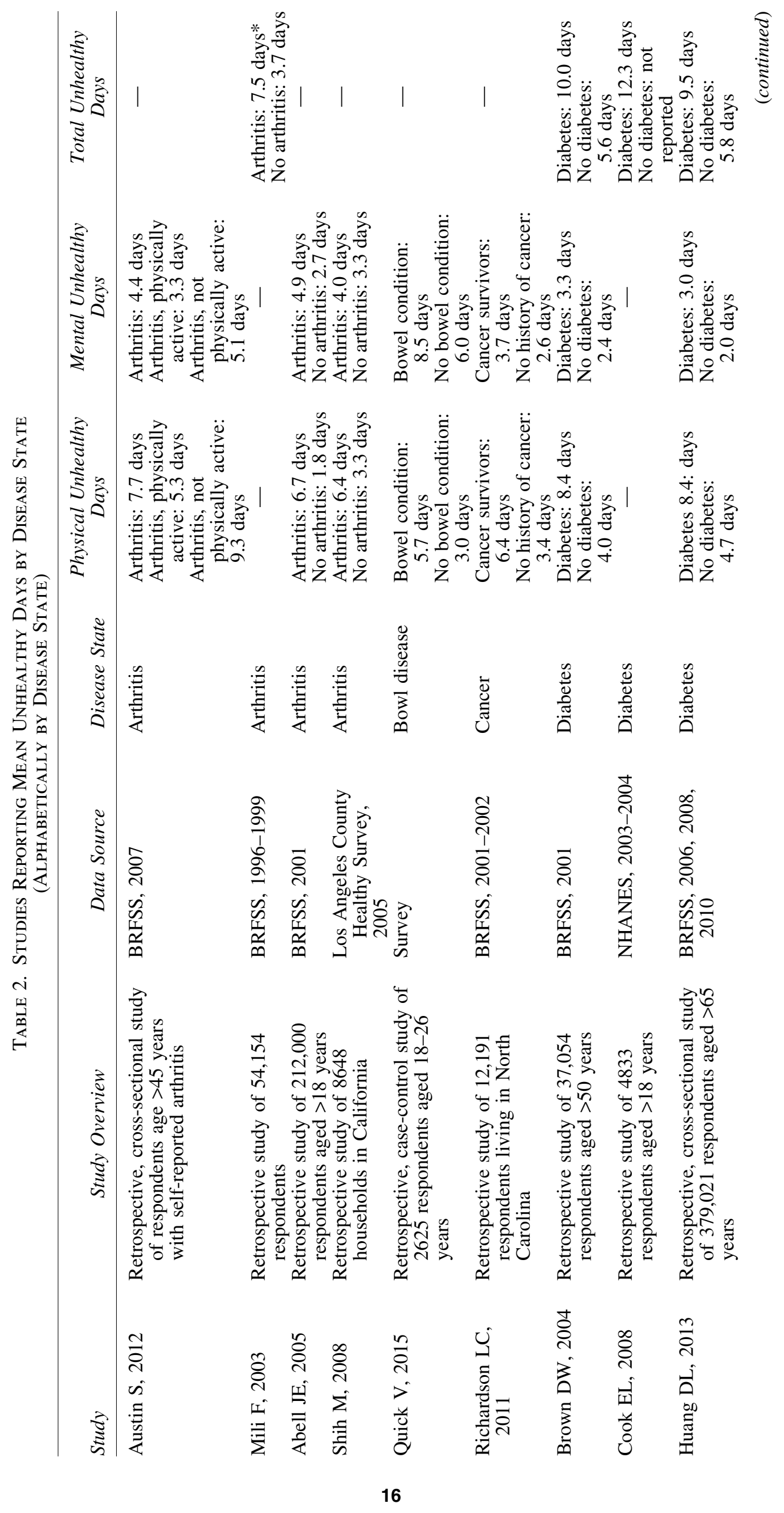




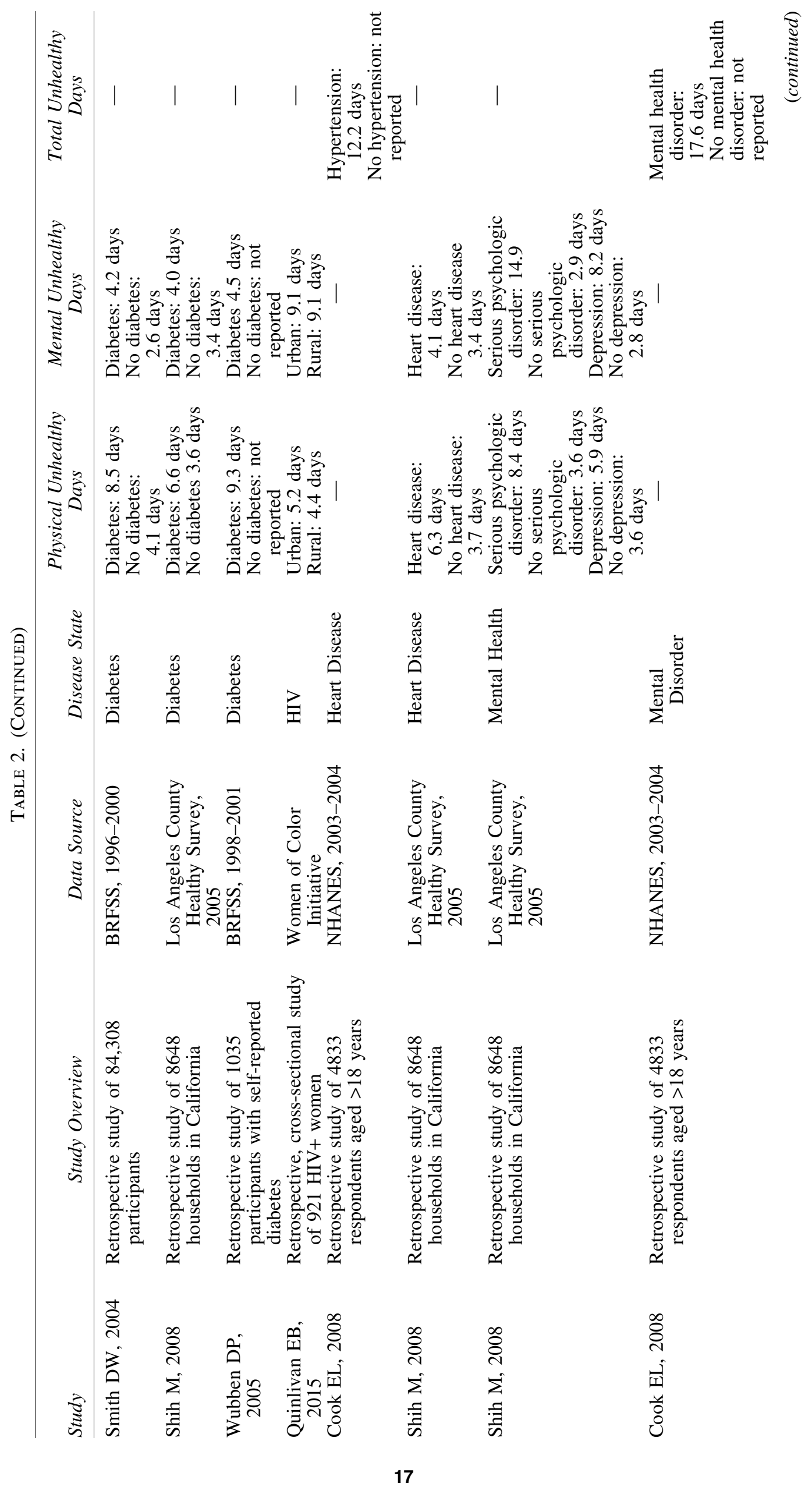




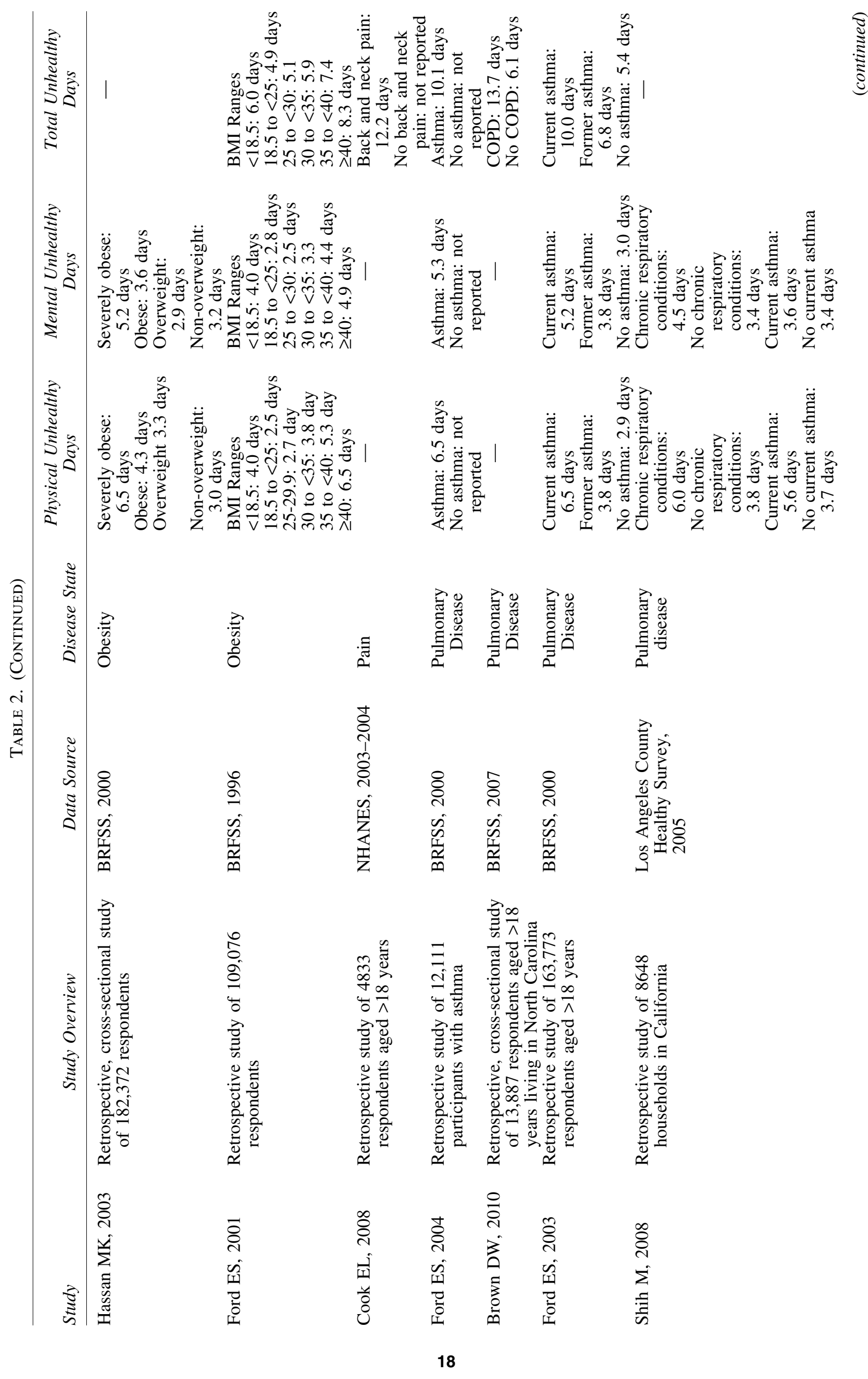




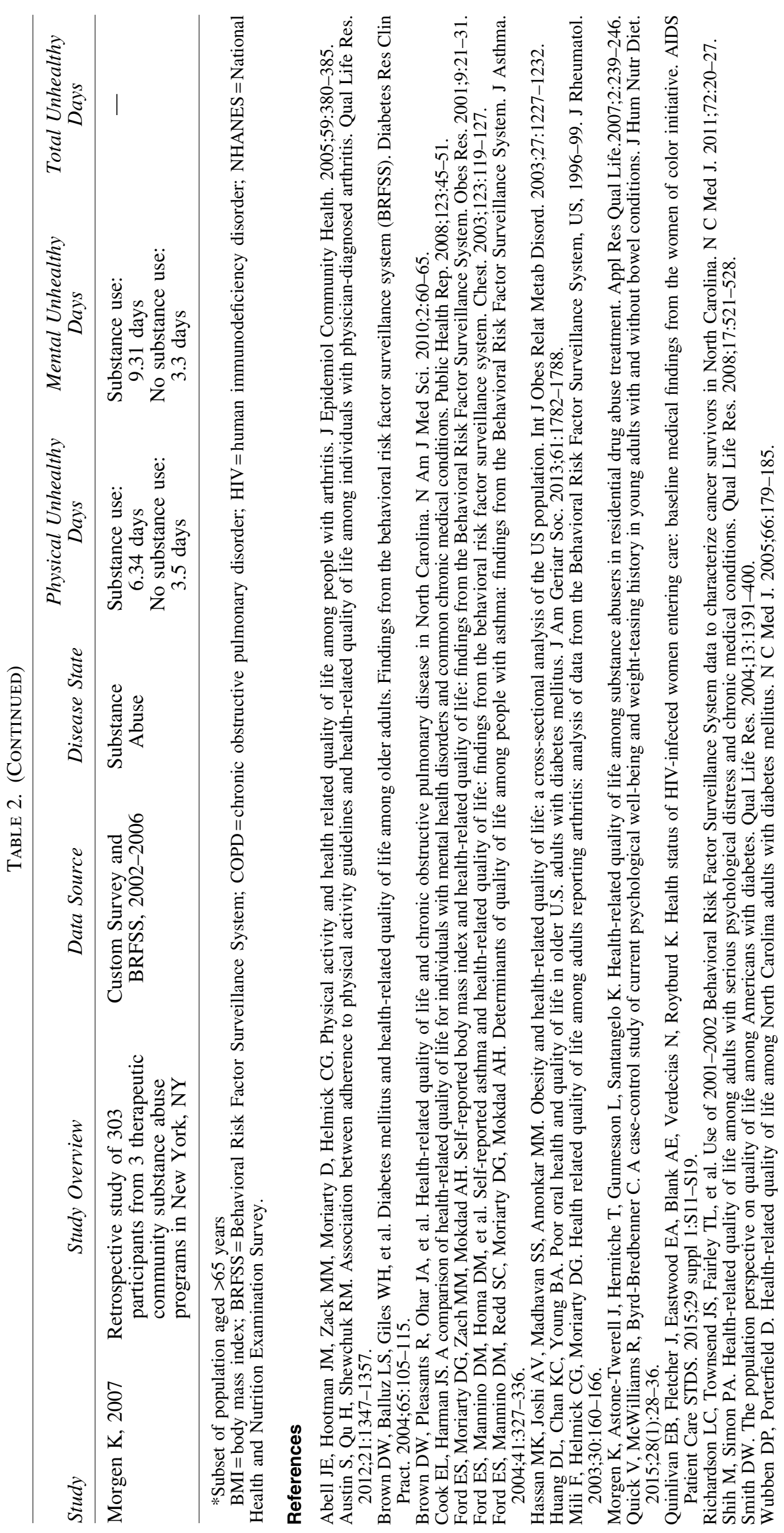


Adjustments for differential sampling probabilities driven by stratified random sampling and adjustment for nonresponse must be considered for sample surveys so that aggregate results can be considered representative of the underlying population. Adjustments for oversampling typically present little issue when the base population is known. In this case, each participant's response can be weighted according to the relative size, in the underlying population, of the stratum (subgroup) to which that participant belongs. However, adjustments for nonresponse can present a larger challenge. If survey nonresponse within each stratum is associated with particular participant characteristics, then further adjustment, or reweighting, is necessary after adjustment for oversampling so that the reweighted survey results are fully applicable to the underlying population. Iterative proportional fitting is the technique currently used in the BRFSS survey to adjust for nonresponse. Numerous additional methods can be considered to adjust for nonresponse including poststratification, such as weighting using logistic regression and replicate weighting. Ultimately the decision is driven by available data for the survey sample and the target population, in addition to the volume of the observed differences. Data necessary for weighting may have to be drawn from outside sources. For example, organizations conducting surveys in Medicare populations can obtain data such as race/ethnicity or income from the Centers for Medicare \& Medicaid Services (CMS).

Once results have been weighted, researchers need to consider several factors in analyzing Healthy Days data. Mean UHD values and percentages for response categories for the target population can be estimated along with $95 \%$ confidence intervals accounting for the survey design using variance estimation techniques now available in most standard statistical software packages.

As an alternative to a mean value for each Healthy Days measure, many researchers construct "total" UHD as a sum of mentally UHD and physically UHD. Although the calculated sum of UHD can exceed 30 days, most investigators cap this sum at 30 days for purposes of interpretability. However, capping in this way can be misleading. Capping precludes identification of those with the poorest health (ie, those with the equivalent of more than 30 overall UHD). As an example, the response of 15 physically UHD and 16 mentally UHD implies an overlap of between 1 day (minimal overlap) and 15 days (maximal overlap). In other words, the respondent experienced anywhere from 1 to 15 days that were both physically and mentally unhealthy and 1 day that was only mentally unhealthy. A cap of 30 would not reflect the fact that at least 1 day was characterized by both physical and mental poor health.

For more complex analysis of the factors associated with $\mathrm{UHD}$, different forms of multivariate regression allow researchers to simultaneously take into account numerous factors and to estimate the degree of impact for each factor. Generalized linear modeling (GLM) using ordinary least squares regression has been used in several published studies to examine mean UHD. However, each Healthy Days question has a highly skewed distribution, with zero being the most common response in most populations. Because of the nature of the question requiring recall, many respondents report Healthy Days in intervals of 5. The resulting distribution has peaks and therefore may violate the assumption of normal residuals in the GLM. Converting UHD data into a binary (frequently $<14$ $\mathrm{UHD}$ or $\geq 14 \mathrm{UHD}$ ), ordinal, or categorical variable to perform logistic, multinomial, or ordinal regression can be a helpful way to relax assumptions regarding the distribution of UHD in continuous form. Many popular software packages today include a survey GLM and logistic regression procedures, making these an attractive option.

Less well-known modeling techniques also can be considered. One option is the zero-inflated negative binomial (ZINB) regression model, which was developed for count variables with excessive zero-value observations. Although one published study has shown the skewed nature of Healthy Days to fit well to the ZINB ${ }^{25}$ none of the popular software packages (at the time of writing) have this capability built in for survey data. The ZINB model also is complicated to communicate, and makes several underlying assumptions that do not apply to UHD; for example, the data range from zero to infinity. Additionally, ZINB does not present a uniform solution, as some subpopulations, particularly those with disability or chronic disease, will not present with the excess zeroes seen in healthier populations. Tobit, Spline and censored least absolute deviations models make no assumptions regarding the underlying distribution and both are commonly used to analyze HRQOL scores, particularly in studies with censored/truncated data or data with strong ceiling/floor effects. ${ }^{26-28}$ The type of censoring addressed by these models occurs with use of a scale in which all values above or below a threshold value are counted as equal to the threshold. Ceiling/floor effects occur when the bulk of values are at either end of the scale, which would be the case in very healthy or very unhealthy populations. Although these models have not been used in the published literature to analyze Healthy Days, their use is plausible as the scores can be considered censored at zero and 30 days and responses are often skewed toward zero. However, few software packages have these methods available.

An alternative modeling approach is use of regression methodologies that do not make assumptions regarding the underlying distribution. The bootstrap method provides results that are at once robust and easy to explain. ${ }^{29}$ Bootstrapping is a nonparametric approach that uses simulated resampling of the study sample and recalculation of the estimate many times to replace the traditional distributional assumptions when calculating a confidence interval or other measure of the variance in estimate. Complications related to the survey design can be accounted for using estimates from survey regression procedures as the basis of the simulation. One shortcoming that may be encountered in more complex regression models such as bootstrapping is their computationally intensive nature, which can slow the modeling process if multiple simulations are required.

If variance estimation is less important and the analyst is primarily concerned with understanding what factors are associated with increased or decreased UHD, decision tree regression provides an additional alternative for analysis without assumptions regarding underlying distribution. In decision tree regression, a large number of factors with potential association with UHD can be considered simultaneously and ranked according to their prevalence in the population and the extent to which they differentiate between individuals with more or fewer UHD. For trended analysis, structured time-series autoregressive integrated moving average modeling has been used successfully to highlight the seasonal effect associated with Healthy Days data while accounting for worsening physical and mental health over time. ${ }^{30}$ 
As with all instruments, the Healthy Days survey instrument has limitations that should be noted. First, the instrument was validated for each question individually. Thus, although the sum of mentally UHD and physically UHD is commonly used to summarize overall health in a single value, this application has not yet been fully validated. Furthermore, unlike other preference-based HRQOL measures such as EQ5D and SF-6D, total UHD cannot be used directly to calculate quality-adjusted life-years (QALYs). However, regression analysis using physically and mentally UHD as explanatory variables can be used to predict measures like the EQ-5D, which can in turn be converted to QALYs for cost-utility studies. ${ }^{31,26}$ Another limitation is that Healthy Days has not been validated in languages other than English. Syntax and diction varies between languages. Non-English speakers can potentially respond differently to a translated HRQOL-4, especially the first question assessing general health. Lastly, wording changes and question order could affect responses; thus, enforcing the standardization of HRQOL-4 is important.

\section{Using Healthy Days to Inform Public Policy}

The Institute of Medicine's 2012 Living Well with Chronic Illness report concluded that it is critical to implement new public policies to promote living well with chronic illnesses. ${ }^{32}$ Accordingly, the Healthy Days measures have broad applications for federal, state, and local governments to better understand the needs of their communities and to identify vulnerable subpopulations. Several groups are using Healthy Days to inform such policy efforts. For more than a decade, CMS has included the Healthy Days questions in the Medicare Health Outcomes Survey, a longitudinal patient-reported outcomes tool used to measure the quality of care provided by Medicare Advantage organizations. ${ }^{33}$ The National Women's Law Center also uses this measure in its report card on women's health to advocate on behalf of women's health and corresponding policy blueprints. ${ }^{34}$ Similarly, the University of Wisconsin's Mobilizing Action Toward Community Health (MATCH) project uses the Healthy Days measures in its county-level health rankings and uses the information to implement programs and policies addressing community health needs. ${ }^{35}$

Now more than ever, the health care system is focused on finding ways to improve well-being from the limited resources available. In January of 2015, US Secretary of Health and Human Services Sylvia M. Burwell announced a goal to have $50 \%$ of all Medicare payments tied to quality or value through alternative payment models by the end of $2018 .{ }^{36}$ As the health care systems shifts to a value-based purchasing model, individuals' perceptions of their health are a critical element of defining value. Patient-reported assessments of their health such as Healthy Days should play an important role in determining where value is being delivered and should be reflected in reimbursement models. Accurate, reliable, and consistent measurement of quality of life using a validated measure like Healthy Days will help to identify promising payment solutions going forward.

Given that quality of life is closely associated to measurable health outcomes, policy makers may find Healthy Days a powerful tool for predicting the needs and use of health care in a community. Expanded utilization of Healthy Days measures as an indicator of the current perceived health of communities could help health planners and leg- islators allocate resources for health services and assess impact of population-based policies or interventions.

\section{Opportunity for Broader Use of Healthy Days}

The Healthy Days survey instrument offers several strengths for measuring population health:

- has demonstrated validity and reliability,

- incorporates the individual's current perspective of health and well-being,

- is easy to administer and respond to, allowing for large population-based surveys,

- produces interpretable and meaningful results that are easily understood by policy makers and the public,

- is correlated to health outcomes, and

- can be tracked over time and compared to national and state benchmarks.

Given these merits, several organizations with important roles in managing and improving population health have adopted Healthy Days as a measurement instrument. Such entities are actively using the data collected to advocate on behalf of the health care needs of their communities. Despite these adopters, great opportunity remains across a wide range of settings for those who manage population health to leverage the Healthy Days tool to track and improve health within their settings. In this time of exponential growth in biometric data collection and the digitization of health records, the addition of data related to the individual's perception of health and well-being fills an important gap in what can be gathered from electronic health records and billing data. Such a holistic view can more accurately isolate the most pressing health care needs of a population, measure improvements resulting from interventions that may otherwise take years to realize the outcomes benefits, and allocate the provision of health care services toward those that improve not only outcomes, but also quality of life.

\section{Author Disclosure Statement}

The authors declared the following potential conflicts of interest with respect to the research, authorship, and/or publication of this article: Drs. Davidson, Happe, Miao, Prewitt, and Slabaugh, Mr. Cordier and Mr. Havens are employees of Humana Inc. Drs. Happe and Slabaugh own Humana stock. No external financial support was provided to Humana for this research. Dr. Jia received compensation from Humana for his contributions. Drs. Shah and Zack report no conflict of interest.

The investigators retained full independence in the conduct of this research. The findings and conclusions in this report are those of the author and do not necessarily represent the official position of the Centers for Disease Control and Prevention.

\section{References}

1. Remington PL, Booske BC. Measuring the health of communities - how and why? J Public Health Manag Pract. 2011;17:397-400.

2. Berwick DM, Nolan TW, Whittington J. The triple aim: care, health, and cost. Health Aff (Millwood). 2008;27:759-769. 
3. Healthy People 2020. Healthy People 2020 Foundation Health Measure Report: Health-Related Quality of Life and WellBeing. November 2010. https://www.healthypeople.gov/sites/ default/files/HRQoLWBFullReport.pdf Accessed November $16,2015$.

4. Moriarty DG, Zack MM, Kobau R. The Centers for Disease Control and Prevention's Healthy Days Measures-population tracking of perceived physical and mental health over time. Health Qual Life Outcomes. 2003;1:37.

5. Zahran HS, Kobau R, Moriarty DG, Zack MM, Holt J, Donehoo R. Health-related quality of life surveillance-United States, 1993-2002. MMWR Surveill Summ. 2005;54(4):1-35.

6. DeSalvo KB, Fan VS, Mcdonell MB, Fihn SD. Predicting mortality and healthcare utilization with a single question. Health Serv Res. 2005;40:1234-1246.

7. Latham K, Peek CW. Self-rated health and morbidity onset among late midlife U.S. adults. J Gerontol B Psychol Sci Soc Sci. 2013;68(1):107-116.

8. Schron E, Friedmann E, Thomas SA. Does health-related quality of life predict hospitalization or mortality in patients with atrial fibrillation? J Cardiovasc Electrophysiol. 2014; 25(1):23-28.

9. Dominick KL, Ahern FM, Gold CH, Heller DA. Relationship of health-related quality of life to health care utilization and mortality among older adults. Aging Clin Exp Res. 2002;14:499-508.

10. Idler EL, Benyamini Y. Self-rated health and mortality: a review of twenty-seven community studies. J Health Soc Behav. 1997;38(1):21-37.

11. HealthyPeople.gov. Foundation Health Measures. 2015. http:// www.healthypeople.gov/2020/about/Foundation-HealthMeasures. Accessed December 14, 2015.

12. Centers for Disease Control and Prevention. Measuring Healthy Days: Population Assessment of Health-related Quality of Life. November 2000. http://www.cdc.gov/ hrqol/pdfs/mhd.pdf. Accessed November 16, 2015.

13. Zullig KJ, Valois RF, Huebner ES, Drane JW. Evaluating the performance of the Centers for Disease Control and Prevention core health-related quality of life scale with adolescents. Public Health Rep. 2004;119:577-584.

14. Andresen EM, Fitch CA, McLendon PM, Meyers AR. Reliability and validity of disability questions for US Census 2000. Am J Public Health. 2000;90:1297-1299.

15. Newschaffer CJ. Validation of Behavioral Risk Factor Surveillance System (BRFSS) HRQOL Measures in a Statewide Sample. Atlanta, GA: Centers for Disease Control and Prevention; 1998.

16. Jia H, Lubetkin EI, Moriarty DG, Zack MM. A comparison of Healthy Days and EuroQol EQ-5D measures in two US adult samples. App Res Qual Life. 2007;2:209-221.

17. Andresen EM, Fouts BS, Romeis JC, Brownson CA. Performance of health related quality-of-life instruments in a spinal cord injured population. Arch Phys Med Rehabil. 1999;80:877-884.

18. Ounpuu S, Chambers LW, Chan D, Yusuf S. Validity of the US Behavioral Risk Factor Surveillance System's health related quality of life survey tool in a group of older Canadians. Chronic Dis Can. 2001;22:93-101.

19. Health-related quality of life-Puerto Rico, 1996-2000. MMWR Morb Mortal Wkly Rep. 2002;51(8):166-168.

20. Jia H, Muennig P, Lubetkin EI, Gold MR. Predicting geographical variations in behavioural risk factors: an analysis of physical and mental healthy days. J Epidemiol Community Health. 2004;58:150-155.
21. Stiefel M, Nolan K. Measuring the triple aim: a call for action. Popul Health Manag. 2013;16:219-220.

22. Borawski E, Bowlin S, Wu G, Jia H, Chen H. The use of the Behavioral Risk Factor Surveillance System (BRFSS) in estimating disability at the state and substate levels. Poster presented at: American Public Health Association annual meeting; Indianapolis, IN; 1997:350.

23. Self-reported frequent mental distress among adultsUnited States, 1993-1996. MMWR Morb Mortal Wkly Rep. 1998;47(16):326-331.

24. Segev Z, Arif AA, Rohrer JE. Activity limitations and healthcare access as correlates of frequent mental distress in adults 65 years and older: a Behavioral Risk Factor Surveillance study_2008. J Prim Care Community Health. 2012;3(1):17-22.

25. Zhou H, Siegel PZ, Barile J, et al. Models for count data with an application to Healthy Days measures: are you driving in screws with a hammer? Prev Chronic Dis. 2014;11:E50.

26. Jia H, Zack MM, Moriarty DG, Fryback DG. Predicting the EuroQol Group's EQ-5D index from CDC's “Healthy Days" in a US sample. Med Decis Making. 2011;31:174185.

27. Sullivan PW, Ghushchyan V. Preference-based EQ-5D index scores for chronic conditions in the United States. Med Decis Making. 2006;26:410-420.

28. Li L, Fu AZ. Some methodological issues with the analysis of preference-based EQ-5D index scores. Health Serv Outcomes Res Method. 2009;9:162-176.

29. Freedman D.A. Bootstrapping regression models. Ann Statist. 1981;9:1218-1228.

30. Jia H, Lubetkin EI. Time trends and seasonal patterns of health-related quality of life among U.S. adults. Public Health Rep. 2009;124:692-701.

31. Jia H, Lubetkin EI. Estimating EuroQol EQ-5D scores from population Healthy Days data. Med Decis Making. 2008; 28:491-499.

32. The National Academies of Sciences, Engineering, and Medicine. Report Brief. Living Well with Chronic Illness: A Call for Public Action. January 31, 2012. http://iom.national academies.org/Reports/2012/Living-Well-with-ChronicIllness/Report-Brief.aspx. Accessed November 16, 2015.

33. Centers for Medicare \& Medicaid Services. Medicare Health Outcomes Survey. http://www.hosonline.org/ Accessed November 16, 2015.

34. National Women's Law Center. Making the Grade on Women's Health: A National and State by State Report Card. http://hrc.nwlc.org/. Accessed November 16, 2015.

35. University of Wisconsin Population Health Institute, School of Medicine and Public Health. Mobilizing Action Toward Community Health (MATCH). http://uwphi.pophealth.wisc .edu/programs/match/. Accessed November 16, 2015.

36. US Department of Health and Human Services. Better, Smarter, Healthier: In historic announcement, HHS sets clear goals and timeline for shifting Medicare reimbursements from volume to value. January 26, 2016. http://www.hhs.gov/news/press/2015 pres/01/20150126a.html. Accessed November 16, 2015.

Address correspondence to: Dr. Laura Happe Humana, Inc. 500 W. Main Louisville, KY 40202

E-mail:1happe@humana.com 